\title{
Dithiinmaleimide Functionalized ET Derivatives: Syntheses, Characterization and X-ray Structure
}

\author{
S. Dolder ${ }^{1}$, S-X. Liu ${ }^{1}$, A. Neels ${ }^{2}$, G. Labat ${ }^{2}$, and S. Decurtins ${ }^{1}$ \\ ${ }^{I}$ Departement für Chemie und Biochemie, Universität Bern, Freiestrasse 3, \\ CH-3012 Bern, Switzerland \\ ${ }^{2}$ Institut de Chimie, Université de Neuchâtel, Avenue Bellevaux 51, CH-2007 \\ Neuchâtel, Switzerland
}

Dithiinmaleimide (ethylenedithio)tetrathiafulvalene (1) and N-phenyldithiinmaleimide (ethylenedithio) tetrathiafulvalene (2) have been synthesized from bis(tetraethylammonium) bis(ethylenedithiotetrathiafulvalenyldithiolato)zincate (3) in high yields. Their electrochemical properties were investigated by cyclic voltammetry $(\mathrm{CV})$ measurements which show two reversible redox potentials of the tetrathiafulvalene (TTF) moiety and an irreversible reduction potential of the maleimide ring. The X-ray structure of 1 shows close $S \cdots S$ contacts in the range of the van der Waals radii (3.6 A) and hydrogen bonds between the maleimide unites.

PACS numbers: 81.05.Zx, 81.16.Dn, 82.80.Ej.

\section{INTRODUCTION}

The covalent attachment of metal ion binding groups to bis (ethylenedithio)tetrathiafulvalene (ET) derivatives and their linkage into supramolecular systems has been chosen as a strategy in the elaboration of functional molecular materials with multiphysical properties ${ }^{1,2}$. Due to the $\pi$ $\mathrm{d}$ interaction between the mobile $\pi$-electrons of the organic conducting system and the localized spins on paramagnetic transition metal ions, interesting solid state properties are expected ${ }^{3}$. Dithiinmaleimide-annelated ET derivatives have been chosen as versatile precursors for the preparation of metal coordination compounds. The maleimide function of these precursors itself can be used to coordinate metal ions or can be transformed into carboxylate or imide groups. It should also be possible to use these ET derivatives as building blocks for porphyrins or phthalocyanines with annelated TTF units. In the case of the unsubstituted dithiinmaleimide, oxidation could lead to charge-transfer (CT) complexes with hydrogen bonding interactions ${ }^{4}$. 
Here, the syntheses and characterization of new compounds $\mathbf{1}$ and $\mathbf{2}$, which were prepared from the zincate complex $\mathbf{3}$, are presented (Fig. 1).<smiles>O=c1[nH]c(=O)c2sc3c(sc1=2)SC(=C1SC2=C(SCCS2)S1)S3</smiles><smiles>O=c1c2sc3c(sc=2c(=O)n1-c1ccccc1)SC(=C1SC2=C(SCCS2)S1)S3</smiles>

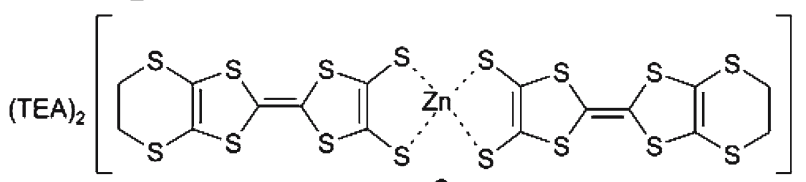

3

Fig. 1. Dithiinmaleimide (ethylenedithio)tetrathiafulvalene 1, N-phenyldithiinmaleimide (ethylenedithio)tetrathiafulvalene $\mathbf{2}$ and zincate complex $\mathbf{3}$

\section{SYNTHESIS}

General information and materials. Compound 3 was prepared according to the literature procedures ${ }^{5}$. All other chemicals and solvents were purchased from commercial sources and were used without any further purification.

Synthesis of Dithiinmaleimide (ethylenedithio)tetrathiafulvalene 1. Compound 3 (700 mg, 3,5 mmol) was suspended in $100 \mathrm{ml}$ degassed $\left(\mathrm{N}_{2}\right)$ acetone. To the dark pink suspension, 2,3-dibromomaleimide $(350 \mathrm{mg}, 7$ mmol) was added. After a few minutes a brownish precipitate was formed and the reaction mixture was stirred overnight. The brownish precipitate was then filtered off and abundantly washed with $\mathrm{MeOH}$ and acetone. Yield: $559.3 \mathrm{mg}(92 \%)$ of brownish 1. Anal. Calcd. for $\mathrm{C}_{12} \mathrm{H}_{5} \mathrm{NO}_{2} \mathrm{~S}_{8} \mathrm{C}, 31.91 ; \mathrm{H}$, 1.12; N, 3.10. Found: C. 31.73; H, 1.24; N, 2.83. ${ }^{1} \mathrm{H}$ NMR (DMSO-d $\left.{ }_{6}\right)$ : $\delta$ $3.41(\mathrm{~s}, 4 \mathrm{H}), 11.57$ (s, 1H). IR (KBr, cm $\left.{ }^{-1}\right)$ 1774, 1708, 1693, 1585, 1338, 1056, 771, 729, 682, 654, 548. Mass spectrum (MALDI): m/z 450.81 (1)

Synthesis of N-phenyldithiinmaleimide (ethylenedithio)tetrathiafulvalene 2. Compound $\mathbf{2}$ was prepared according to $\mathbf{1}$. Instead of 2,3-dibromomaleimide, 2,3-dichloro-N-phenylmalemide was used. The reaction gave brownish 2 in $85 \%$ yield. Anal. Calcd. for $\mathrm{C}_{18} \mathrm{H}_{9} \mathrm{NO}_{2} \mathrm{~S}_{8} \mathrm{C}, 40.96 ; \mathrm{H}, 1.72 ; \mathrm{N}$, 2.65. Found: $\mathrm{C}, 40.86 ; \mathrm{H}, 1.69 ; \mathrm{N}, 2.41 .{ }^{1} \mathrm{H}$ NMR $\left(\mathrm{DMSO}^{-\mathrm{d}_{6}}\right): \delta 3.24(\mathrm{~s}$, 4H), $7.22(\mathrm{~m}, 2 \mathrm{H}), 7.38$ (m, 3H). IR (KBr, cm $\left.{ }^{-1}\right)$ 1775, 1714, 1596, 1502 $1385,1110,774,731,725,689,634,578$. Mass spectrum (MALDI): $\mathrm{m} / \mathrm{z}$ $526.84(2)$

Electrochemical measurements. The solution redox properties of the new compounds $\mathbf{1}$ and $\mathbf{2}$ were investigated in THF by CV. The CV data are presented in Table 1 together with those of ET. 


\begin{tabular}{c|c|c|c}
\hline Compound & $\mathrm{E}_{1}^{1 / 2} / \mathrm{V}$ & $\mathrm{E}_{2}^{1 / 2} / \mathrm{V}$ & $\mathrm{E}_{\text {red }}^{1 / 2} / \mathrm{V}$ \\
\hline $\mathbf{1}^{\mathrm{a}}$ & 0.87 & 0.96 & -0.77 \\
$\mathbf{2}^{\mathrm{a}}$ & 0.87 & 0.95 & -0.80 \\
$\mathrm{ET}^{\mathrm{b}}$ & 0.52 & 0.94 & - \\
\hline
\end{tabular}

Table 1. Cyclic voltammetric data. All oxidation potentials were determined under $\mathrm{N}_{2}$ vs $\mathrm{Ag} / \mathrm{AgCl}$ at room temperature. Measurements have been done at a scan rate of $100 \mathrm{mVs}^{-1}$ using $0.1 \mathrm{M} \mathrm{Bu}_{4} \mathrm{NPF}_{6}$ as electrolyte and $\mathrm{Pt}$ as working electrode. (a) in THF, (b) in $\mathrm{CH}_{2} \mathrm{Cl}_{2}$.

\section{RESULTS AND DISCUSSION}

Contrary to the unsuccessful synthesis of dithiinmalemide ET derivatives from the appropriate 4,5-dithiinemaleimide-1,3-dithiole-2chalcogenones via the trialkylphophite-mediated coupling reaction ${ }^{6}$, two new dithiinmaleimide donors $\mathbf{1}$ and $\mathbf{2}$ were prepared in high yields from the zincate complex 3 .

The crystal packing of compound $\mathbf{1}$ is shown in Figs. 2 and 3. It crystallizes in the triclinic space group P-1 as red blocks.

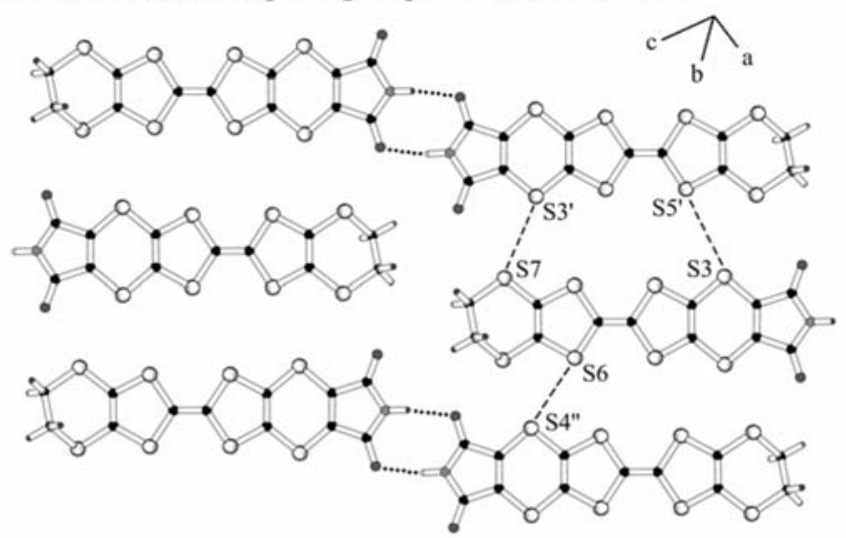

Fig. 2. Layer of head-to-tail arranged molecules of 1 with H-bonds ( $\cdots)$

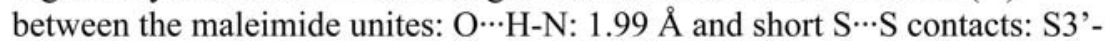

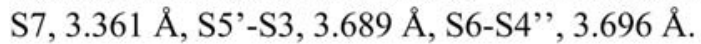

In the CV measurements (Table 1), two typical reversible single-electron redox couples of the TTF unit of $\mathbf{1}$ and $\mathbf{2}$ are observed. Compared to ET, the annelation of the dithiinemaleimide group in $\mathbf{1}$ and 2 increases mainly the first redox potential due to a strong electron-withdrawing effect. At negative potential an irreversible 
reduction peak is also visible, which could be due to the reduction of the maleimide ring to the radical anion as reported for 4,5dithiinmaleimide-1,3-dithiole-2-thione ${ }^{6}$.

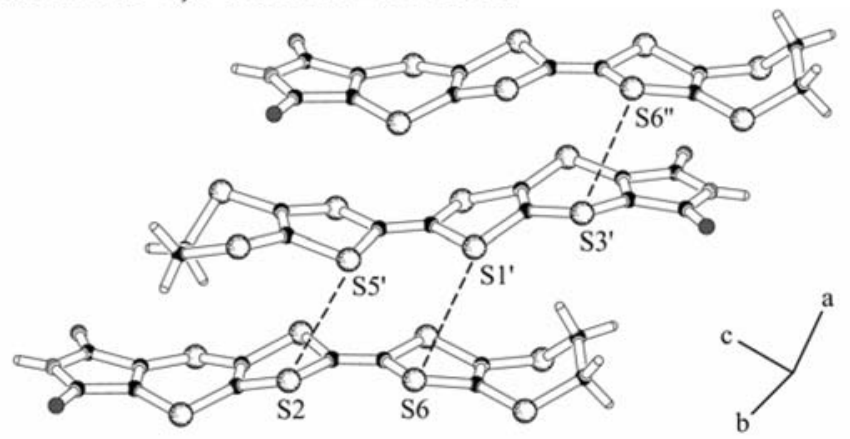

Fig. 3. Short S'-S contacts: S6''-S3', $3.747 \AA$, S1'-S6, $3.794 \AA$, S5'-S2, $3.680 \AA$ between the sheets of bended molecules 1 .

\section{CONCLUSIONS}

The use of the zincate complex 3 has proven to be an effective route to the synthesis of dithiinmaleimide-annelated ET derivatives. Although the prepared compounds show weaker donor abilities than ET, the promising use of $\mathbf{1}$ as versatile building block, for instance for the synthesis of phthalocyanines, will be investigated.

\section{ACKNOWLEDGEMENTS}

This work was supported by the Swiss National Science Foundation (Proj. No. 200020-107589) and the ESF programme SONS (NANOSYN).

\section{REFERENCES}

1. E. Coronado, J. R. Galan-Mascaros, C.J. Gomez-Garcia, Nature 408, 447 (2000)

2. S-X. Liu, S. Dolder, A. Neels, H. Stoeckli-Evans and S. Decurtins, Inorg. Chem. 42, 4801 (2003)

3. L. Ouahab and T. Enoki, Eur. J. Inor. Chem. 5, 933 (2004)

4. Y. Morita, S. Maki, M. Ohmoto, H. Kitagawa, T. Okubo, T. Mitani and K. Nakasuji, Org. Lett. 4, 2185 (2002)

5. K. Ueda, M. Yamanoha, T. Sugimoto, H. Fujita, A. Ugawa, K. Yakushi and K. Kano, Chem. Lett. 5, 461 (1997)

6. T. K. Hansen, S. K. Varma, S. Edge, A. Charlton, A. E. Underhill and J. Becher, J. Chem. Soc. Perkin Trans. 1 14, 1807 (1992) 